\title{
The Difference of the Slope Wind Between Day and Night
}

\author{
By Hiroaki Kondo \\ National Research Institute for Pollution and Resources \\ 16-3, Onogawa, Yatabe, Tsukuba, Ibaraki, 305, Jaban \\ (Manuscript received 3 August 1983, in revised form 10 February 1984)
}

\begin{abstract}
The response of the fluid stably stratified over the infinite slope was investigated by linear theory when finite length $2 l$ in it was heated or cooled. If the angle of the slope was small, the nature of the flow near the slope changed depending on whether the half height of the slope $h_{S}^{*}=l \sin \phi$ ( $\phi$ is angle of slope) was higher than that of thermal boundary layer $h_{T}^{*}=\alpha^{*}(\nu / N)^{1 / 3} l^{1 / 3}(\alpha \sim 3.5)$, which was developed over the heat island whose length was $2 l$, or not.

On the real slope $h_{S}^{*}>h_{T}^{*}$ was often satisfied at night and the flow was close to the results from Prandtl theory, but $h_{S}^{*}<h_{T}^{*}$ in the daytime so that the flow behaved like a convection.
\end{abstract}

\section{Introduction}

One of the most striking characters of topography in Japan is an abundance of mountains, and local winds blown in Japan are more or less affected by these mountains. It was reported by some authors in both observations (Fujibe and Asai, 1979) and numerical experiments (Asai and Mitsumoto (1978), Oukouchi et al. (1978), Sahashi (1981) and Kikuchi et al. (1981)) that land and sea breezes which were the most general feature of local winds caused by the difference of thermal energy released into the atmosphere from the surface were affected by mountains and that they interacted with mountain and valley breezes.

Mannoji (1982) clarified by the numerical experiment that two kinds of breezes were contained in the breezes blown between plain and mountains. That is, slope winds and plain-plateau breezes. Tyson et al. (1972) also pointed out these two breezes by observations in South Africa. In these two breezes Prandtl had considered slope winds by parcel method. This theory explained downslope wind at night fairly well in a valley at mid-latitude and katabatic wind in antarctic but didn't agree with the upslope wind in a valley in the daytime, as pointed out by several authors (for example Sutton, Mannoji etc.). From the obsevation McHattie (1968) concluded that small-scale convections which developed in the daytime brought the effect of upper wind into the valley. But in Mannoji's numerical calculations, even the case without the upper wind, also didn't agree with Prandtl's theory in the daytime. Mannoji concluded that this might be caused by the difference of stability of the atmosphere near the surface between day (neutral-slightly unstable) and night (stable).

Here we investigate the response of stratified fluid by linear theory over the infinite length slope in which finite length is heated or cooled and estimate the difference of the slope wind between day and night with the aid of numerical experiments.

\section{Basic equations}

We consider 2-dimensional rectangular coordinate system $\left(\xi^{*}, \zeta^{*}\right)$ which is inclined against usual $\left(x^{*}, z^{*}\right)$ coordinate system at $\phi$ (Fig. 1).

Then we may represent basic equations as 


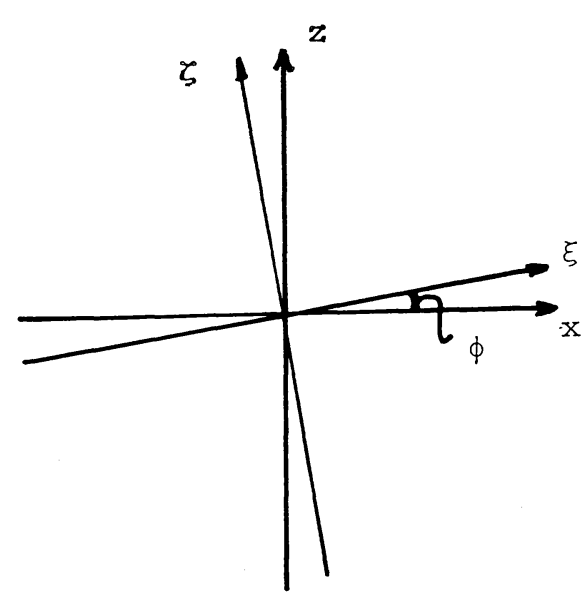

Fig. $1 \xi-\zeta$ coordinate system.

$$
\begin{aligned}
& \frac{\partial u^{*}}{\partial t^{*}}+u^{*} \frac{\partial u^{*}}{\partial \xi^{*}}+w^{*} \frac{\partial u^{*}}{\partial \zeta^{*}} \\
= & -c_{P} \Theta \frac{\partial \hat{\pi}}{\partial \xi^{*}}+\frac{g}{\Theta} \theta^{*} \cdot \sin \phi+\nu \frac{\partial^{2} u^{*}}{\partial \zeta^{2 *}}, \\
& \frac{\partial w^{*}}{\partial t^{*}}+u^{*} \frac{\partial w^{*}}{\partial \xi^{*}}+w^{*} \frac{\partial w^{*}}{\partial \zeta^{*}} \\
= & -c_{P} \Theta \frac{\partial \hat{\pi}}{\partial \zeta^{*}}+\frac{g}{\Theta} \theta^{*} \cdot \cos \phi+\nu \frac{\partial^{2} w^{*}}{\partial \zeta^{2 *}}, \\
& \frac{\partial \theta^{*}}{\partial t^{*}}+u^{*} \frac{\partial \theta^{*}}{\partial \xi^{*}}+w^{*} \frac{\partial \theta^{*}}{\partial \zeta}=\kappa \frac{\partial^{2} \theta^{*}}{\partial \zeta^{2 *}}, \\
& \frac{\partial u^{*}}{\partial \xi^{*}}+\frac{\partial w^{*}}{\partial \zeta^{*}}=0,
\end{aligned}
$$

under Boussinesq and anelastic approximations. Here $\left(u^{*}, w^{*}\right)$ are $\left(\xi^{*}, \zeta^{*}\right)$ components of velocity, $\hat{\pi}=\left(p^{*} / P\right)^{R / C} P$, where $p^{*}$ is pressure, $P$ is a reference pressure, $R$ is gas constant and other symbols have usual meanings (The variables with $*$ are dimensionalized forms.). We assume $\kappa=\nu$, for turbulent diffusivity of heat is nearly equal to that of momentum in most cases of usual atmosphere. For potential temperature

$$
\begin{aligned}
\theta^{*}\left(\xi^{*}, \zeta^{*}, t^{*}\right) & =\Theta_{z}+\bar{\theta}_{z}^{*}\left(z^{*}\right)+\theta_{z}^{*}\left(x^{*}, z^{*}, t^{*}\right) \\
& =\Theta+\bar{\theta}^{*}\left(\xi^{*}, \zeta^{*}\right)+\theta^{*}\left(\xi^{*}, \zeta^{*}, t^{*}\right),
\end{aligned}
$$

where $\bar{\theta}^{*}\left(\xi^{*}, \zeta^{*}\right)=\gamma\left(\xi^{*} \cdot \sin \phi+\zeta^{*} \cdot \cos \phi\right)$.

As a basic state we consider static state which is stably stratified in vertical $(z)$ direction such as $\partial \bar{\theta}_{z}^{*} / \partial z^{*}=\gamma$ (constant). Then square of bouyancy frequency is given by $N^{2}=(g / \Theta) \gamma$.
Here we consider the motion near origin so that $\xi^{*} \cdot \gamma \sin \phi, \zeta^{*} \cdot \gamma_{i}^{*} \cos \phi \ll \Theta$.

We describe perturbation field with $\left(^{\prime}\right)$ and basic field with (-) then equations for the basic field are reduced to

$$
\begin{aligned}
& c_{P} \Theta \frac{\partial \overline{\hat{\pi}}}{\partial \xi^{*}}=\frac{g}{\Theta} \bar{\theta}^{*} \sin \phi, \\
& c_{P} \Theta \frac{\partial \overline{\bar{\pi}}}{\partial \zeta^{*}}=\frac{g}{\Theta} \bar{\theta}^{*} \cos \phi,
\end{aligned}
$$

and these mean hydrostatic equilibrium. For the perturbation field we neglect higher order terms than 1st order, the basic equations become

$$
\begin{aligned}
& \frac{\partial u^{* *}}{\partial t^{*}}=-c_{P} \Theta \frac{\partial \hat{\pi}^{\prime}}{\partial \xi^{*}}+\frac{g}{\Theta} \theta^{* *} \sin \phi+\nu \frac{\partial^{2} u^{* *}}{\partial \zeta^{2 *}} \\
& \frac{\partial w^{* *}}{\partial t^{*}}=-c_{P} \Theta \frac{\partial \hat{\pi}^{\prime}}{\partial \zeta^{*}}+\frac{g}{\Theta} \theta^{\prime *} \cos \phi+\nu \frac{\partial^{2} w^{\prime *}}{\partial \zeta^{2 *}} \\
& \frac{\partial \theta^{\prime *}}{\partial t}+\frac{\Theta}{g} N^{2}\left(u^{\prime *} \sin \phi+w^{* *} \cos \phi\right)=\nu \frac{\partial^{2} \theta^{* *}}{\partial \zeta^{2 *}} \\
& \frac{\partial u^{* *}}{\partial \xi^{*}}+\frac{\partial w^{*}}{\partial \zeta^{*}}=0 .
\end{aligned}
$$

Next, equations (5)-(8) are nondimensionalized as follows :

$$
\begin{array}{ll}
\left(\xi^{*}, \zeta^{*}\right)=(l \xi, l \zeta), & t^{*}=t N^{-1}, \\
\left(u^{*}, w^{*}\right)=\left(l N u^{\prime}, l N w^{\prime}\right), & c_{P} \Theta \hat{\pi}^{\prime}=\frac{l^{3} N^{4}}{g} \pi^{\prime}, \\
\theta^{*}=\Theta \theta^{\prime} . &
\end{array}
$$

Here $l$ is a half of the slope length in which heating and/or cooling are given. Then nondimensionalized equations are

$$
\begin{gathered}
\frac{\partial u^{\prime}}{\partial t}=-\delta \frac{\partial \pi^{\prime}}{\partial \xi}+\delta^{-1} \theta^{\prime} \sin \phi+R^{-1 / 2} \frac{\partial^{2} u^{\prime}}{\partial \zeta^{2}}, \\
\frac{\partial w^{\prime}}{\partial t}=-\delta \frac{\partial \pi^{\prime}}{\partial \zeta}+\delta^{-1} \theta^{\prime} \cos \phi+R^{-1 / 2} \frac{\partial^{2} w^{\prime}}{\partial \zeta^{2}},
\end{gathered}
$$

$$
\frac{\partial \theta^{\prime}}{\partial t}+\delta\left(u^{\prime} \sin \phi+w^{\prime} \cos \phi\right)=R^{-1 / 2} \frac{\partial^{2} \theta^{\prime}}{\partial \zeta^{2}},
$$




$$
\frac{\partial u^{\prime}}{\partial \xi}+\frac{\partial w^{\prime}}{\partial \zeta}=0
$$

Here $\delta^{-1}=g / N^{2} l, R^{-1 / 2}=\nu / N l^{2}$.

First we investigate the phenomena led from (9)-(12) under several idealized conditions and then analyze the nature of the flow over the slope with finite heat and/or cool source.

\section{Some features of stratified fluid obtained from reduced characteristic equation}

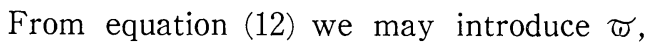
where

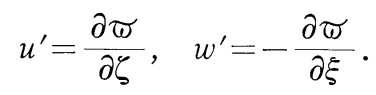

Then equations (9)-(12) become

$$
\begin{aligned}
& \left(\frac{\partial}{\partial t}-R^{-1 / 2} \frac{\partial^{2}}{\partial \zeta^{2}}\right)\left(\frac{\partial \theta^{\prime}}{\partial \xi}\right) \\
& =\delta\left(\cos \phi \cdot \frac{\partial}{\partial \xi}-\sin \phi \cdot \frac{\partial}{\partial \zeta}\right)\left(\frac{\partial \varpi}{\partial \xi}\right), \\
& \left(\frac{\partial}{\partial t}-R^{-1 / 2} \frac{\partial^{2}}{\partial \zeta^{2}}\right)\left(\frac{\partial^{2}}{\partial \xi^{2}}+\frac{\partial^{2}}{\partial \zeta^{2}}\right)\left(\frac{\partial \varpi}{\partial \xi}\right) \\
& =-\delta^{-1}\left(\cos \phi \cdot \frac{\partial}{\partial \xi}-\sin \phi \cdot \frac{\partial}{\partial \zeta}\right)\left(\frac{\partial \theta^{\prime}}{\partial \xi}\right) .
\end{aligned}
$$

From (13) and (14) we have

$$
\begin{aligned}
& {\left[\left(\frac{\partial}{\partial t}-R^{-1 / 2} \frac{\partial^{2}}{\partial \zeta^{2}}\right)^{2}\left(\frac{\partial^{2}}{\partial \xi^{2}}+\frac{\partial^{2}}{\partial \zeta^{2}}\right)\right.} \\
& \left.\quad+\left(\cos \phi \cdot \frac{\partial}{\partial \xi}-\sin \phi \cdot \frac{\partial}{\partial \zeta}\right)^{2}\right] \Phi=0,
\end{aligned}
$$

where $\Phi$ is $\partial \theta^{\prime} / \partial \xi$ or $\partial \varpi / \partial \xi$.

Here we assume that $\theta^{\prime}$ and $\varpi$ have next forms :

$$
\theta^{\prime}(\omega, k, n ; \xi, \zeta, t)=e^{i \omega t} e^{i k \xi} e^{n \zeta} \hat{\theta}(\omega, k, n),
$$

$\widetilde{\varpi}(\omega, k, n ; \xi, \zeta, t)=e^{i \omega t} e^{i k \xi} e^{n \zeta} \hat{\widetilde{\omega}}(\omega, k, n)$.

These froms correspond to homogeneous wavelike solutions about time and $\xi$ coordinates.

Substitute (16), (17) into (15) then we have $\left(i \omega-R^{-1 / 2} n^{2}\right)^{2}\left(n^{2}-k^{2}\right)+(i k \cos \phi-n \sin \phi)^{2}$ $=0$.
Some ideal features of stratified fluid are derived by reducing equation (15) or (18).

\subsection{Steady state with $\phi=0$}

In this case (15) is reduced to

$$
\left[\frac{\partial^{4}}{\partial \zeta^{4}}\left(\frac{\partial^{2}}{\partial \xi^{2}}+\frac{\partial^{2}}{\partial \zeta^{2}}\right)+R \frac{\partial^{2}}{\partial \xi^{2}}\right] \Phi=0 .
$$

This corresponds to steady convection mode. When slab symmetric boundary condition given for $\theta^{\prime}$, the solution represents a heat island. If we expect to seek the solution which satisfies $\partial^{2} / \partial \zeta^{2} \gg \partial^{2} / \partial \xi^{2}$ (i.e. the aspect ratio of the phenomenon is very small),

$$
\left[\frac{\partial^{6}}{\partial \zeta^{6}}+R \frac{\partial^{2}}{\partial \xi^{2}}\right] \Phi=0
$$

This corresponds to the convection of Stommel and Veronis (1957). If we introduce a vertical scale length $h(h \ll 1)$ for the perturbation with horizontal scale length 2 , then

$$
h^{6}=R^{-1} \text {. }
$$

Next we seek the solution which satyisfy $\partial^{2} / \partial \zeta^{2} \ll \partial^{2} / \partial \xi^{2}$ (i.e. the aspect ratio of the phenomenon is very large), then

$$
\left(\frac{\partial^{4}}{\partial \zeta^{4}} \cdot \frac{\partial^{2}}{\partial \xi^{2}}+R \frac{\partial^{2}}{\partial \xi^{2}}\right) \Phi=0
$$

and

$$
h \sim R^{-1 / 4} .
$$

In dimensional form $h^{*} \sim(\nu / N)^{1 / 2}$, that is, $h^{*}$ doesn't depend on horizontal scale of heat source.

\subsection{Non steady state with $\phi=0$}

In this case from (15) we obtain

$$
\left[\left(\frac{\partial}{\partial t}-R^{-1 / 2} \frac{\partial^{2}}{\partial \zeta^{2}}\right)^{2}\left(\frac{\partial^{2}}{\partial \xi^{2}}+\frac{\partial^{2}}{\partial \zeta^{2}}\right)+\frac{\partial^{2}}{\partial \xi^{2}}\right] \Phi=0 .
$$

In case of $\partial^{2} / \partial \zeta^{2} \gg \partial^{2} / \partial \xi^{2}$, Kimura and Eguchi (1978) introduced this as land and sea breezes. If $\partial / \partial t \sim R^{-1 / 2}\left(\partial^{2} / \partial \zeta^{2}\right)$, vertical structure of the phenomenon is affected by the periodic change of boundary condition of $\theta^{\prime}$. That is, there is a horizontal scale of the heat source over which the development of the boundary layer as $l$ becomes larger is suppressed by the periodic change of the boundary condition for 
$\left.\theta^{\prime} .1\right)$

\subsection{Non steady state with $\phi \neq 0$}

\subsection{1 $R \gg 1$}

Here we consider characteristic equation (18). In this case (18) is reduced to

$$
-\omega^{2}\left(n^{2}-k^{2}\right)+(i k \cos \phi-n \sin \phi)^{2}=0,
$$

i.e. $\left(\sin ^{2} \phi-\omega^{2}\right) n^{2}-2 i k n \cos \phi \cdot \sin \phi$

$$
+k^{2}\left(\omega^{2}-\cos ^{2} \phi\right)=0 \text {. }
$$

This is dispersion relation of internal gravity wave in $\xi-\zeta$ coordinate system. $R \gg 1$ means $\nu \ll N l^{2}$. For example, in case of weak diffusion (small turbulent viscosity) the dominant phenomenon is internal gravity wave or in case of not so weak diffusion the dominant phenomenon except very near the surface is also internal gravity wave, if there are external forces to make perturbations.

3.3.2 $\partial^{2} / \partial \zeta^{2} \gg \partial^{2} / \partial \xi^{2}$ with not small $\phi$

Here returning to (15), the equation is reduced to

$$
\left[\left(\frac{\partial}{\partial t}-R^{-1 / 2} \frac{\partial^{2}}{\partial \zeta^{2}}\right)^{2}+\sin ^{2} \phi\right] \Phi=0 .
$$

This case was introduced in Asai and Mitsumoto (1978) as slope winds with periodic change of boundary condition. If $\partial / \partial t \ll \sin \phi$ (If periodic change with frequency $\omega$ is assumed, $\omega \ll \sin \phi$.$) , and this is true in most$ cases of slope winds in the field as pointed out by Asai and Mitsumoto, (20) becomes,

$$
\left[R^{-1 / 2} \frac{d^{2}}{d \zeta^{2}} \pm i \sin \phi\right] \Phi=0 \text {. }
$$

This corresponds to the results obtained by Prandtl (1942). In case of $\phi=0$ in (20),

$$
\frac{\partial}{\partial t} \Phi=R^{-1 / 2} \frac{\partial^{2}}{\partial \zeta^{2}} \Phi
$$

This case was introduced in Kimura and Eguchi (1978) as conduction wave.

3.3.3 $\partial^{2} / \partial \zeta^{2} \ll \partial^{2} / \partial \xi^{2}$ with not small $\phi$

In this case from (15)

1) Kimura and Eguchi (1978) pointed out that there was a scale over which the height of land and sea breezes could not 'feel' the scale of heat source (about $100 \mathrm{~km}$ ).

$$
\left[\left(\frac{\partial}{\partial t}-R^{-1 / 2} \frac{\partial^{2}}{\partial \zeta^{2}}\right)^{2}+\cos ^{2} \phi\right] \Phi=0 .
$$

The aspect ratio of disturbance is very large and this case may not exist in real field.

If we choose parameters given in Table 1 for (18) with the conditon of $\omega=0$, real part of three solutions for $n$ out of six solutions. in (18) are not positive. These three solutions are close to each other when $\phi=0$, but as $\phi$ increases, two of them approach to Prandtl's case and another approaches the internal gravity wave (stationary) case (i.e. $\operatorname{Re}(n) \rightarrow 0$ in Fig. 2).

Table 1 Parameters used in equation (18) for the calculation of Fig. 2.

$$
\begin{aligned}
l: & 100 \mathrm{~km} \\
N^{2}: & 1.96 \times 10^{-4} \mathrm{~s}^{-2} \\
\nu: & 10 \mathrm{~m}^{2} \mathrm{~s}^{-1} \\
k: & 2 \pi \\
\omega: & 0
\end{aligned}
$$

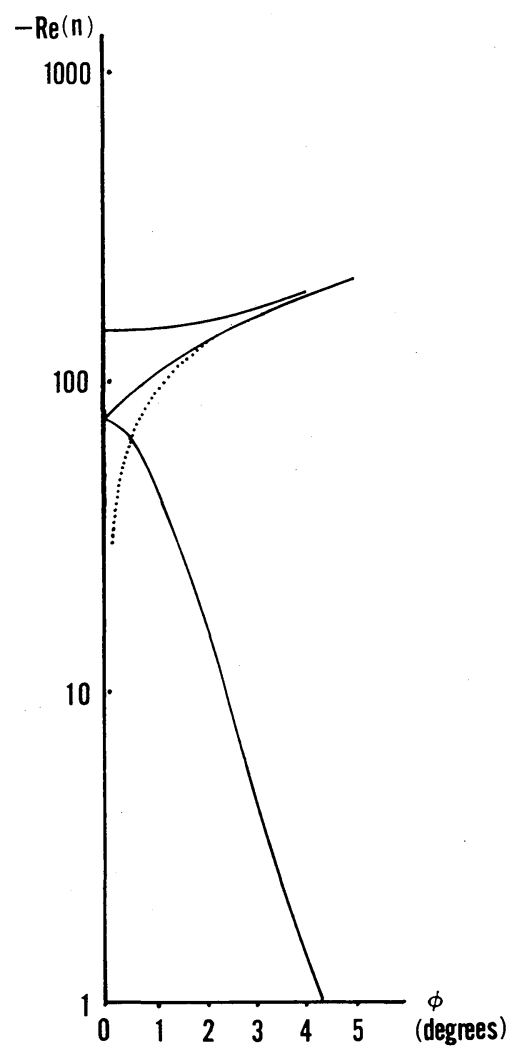

Fig. 2 Dependency of the real parts of three solutions (solid lines) of (18) on $\phi$ (with $\omega=0)$. Parameters are given in Table 1. Dotted line is obtained from Prandtl's theory. 


\section{Slope winds over the finite heat source}

Here we consider the air flow caused by a heat source of finite length (The length is 2.) on the infinite slope. Then boundary conditions are,

$$
\begin{aligned}
& u^{\prime}, w^{\prime}, \theta^{\prime}<\infty \quad \text { as } \zeta \rightarrow \infty, \\
& u^{\prime}, w^{\prime}=0 \quad \text { at } \zeta=0, \\
& \theta^{\prime}(\xi, 0, t)=e^{i \omega t} \frac{2 \theta_{0}}{\pi} \int_{0}^{\infty} \cos (k \xi) \frac{\sin k}{k} d k .
\end{aligned}
$$

If we assume that $\tilde{\theta}(\omega, k, n ; \xi, \zeta, t)$ is the solution of (15) for $(\omega, k, n)$ mode, then

$$
\begin{aligned}
& \theta^{\prime}(\xi, \zeta, t)=\frac{1}{\pi} e^{i \omega t} \int_{0}^{\infty} \frac{\sin k}{k}\{\hat{\theta}(k, n(k) ; \xi, \zeta) \\
& \quad+\hat{\theta}(-k, n(-k) ; \xi, \zeta)\} d k
\end{aligned}
$$

where $\tilde{\theta}(\omega, k, n(k) ; \xi, \zeta, t)=e^{i \omega t} \hat{\theta}(k, n(k) ; \xi, \zeta)$,

$$
\hat{\theta}(k, n(k) ; \xi, 0)=\theta_{0} e^{i k \xi},
$$

and $\hat{\theta}(-k, n(-k) ; \xi, 0)=\theta_{0} e^{-i k \xi}$.

Here after we consider stationary case $(\omega=0)$ for the sake of simplicity.

If $\phi=0$, these boundary conditions mean a heat island. In case of $\phi \neq 0$ the phenomena contain not only heat island but also slope wind. We must separate these two phenomena to investigate the nature of slope wind. These two phenomena may be separated when we consider that the stream-function of heat island is an antisymmetric perturbation field about $\zeta$ axis. Under Boussinesq approximation, this antisymmetry of heat island may approximately hold in case of $\phi \neq 0$ for small $\phi$. Then the perturbation field from which we subtract antisymmetric part may approximately represent the slope wind. That is, the motion on the $\zeta$ axis may be ascribed to the slope wind. Fig. 3 shows streamlines for $R^{1 / 4}=1000$. (a), (b) and (c) are $\phi=0^{\circ}, \phi=0.5^{\circ}$ and $\phi=4^{\circ}$, respectively (These figures still contain the two features discussed above.). In case of (a) streamlines are symmetric about $\zeta$ axis and two cells of convection are clear. In case of (b), this is an under-critical situation (see below), there are still two cells but left one is developed and elongated. The streamlines away from the surface become straight. In case of (c), this is a super critical situation,

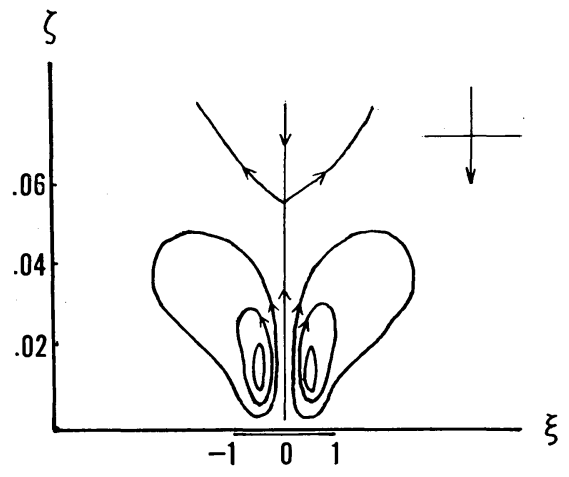

(a)

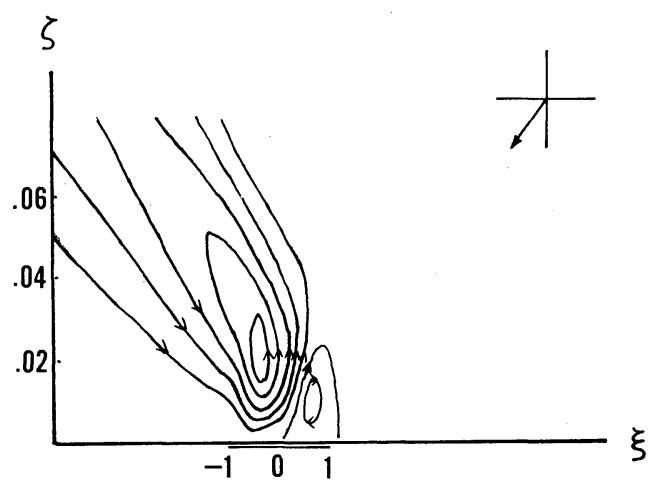

(b)

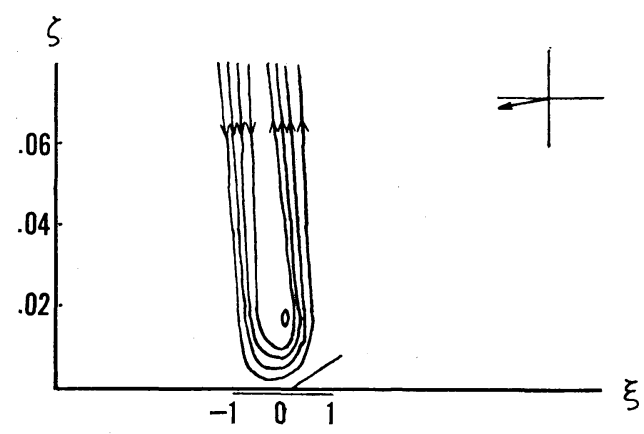

(c)

Fig. 3 The streamlines over the infinite slope with finite heat source for $R^{1 / 4}=1000$. (a) $\phi=0^{\circ}$, (b) $\phi=0.5^{\circ}$, and (c) $\phi=4^{\circ}$. An arrow at upper right corner shows the direction of the gravity in the figure.

there is only one cell and the cell is elongated more than in case (b).

Now we pay attention two features of the results obtained by the solutions, the maximum velocity $u_{\max }^{*}$ and the height $\left(h_{u}\right)$ where $u_{\max }^{*}$ is given on the $\zeta$ axis, and investigate $\phi$-dependency of these two parameters. Fig. 4 
shows the relation between $u_{\max }^{*}$ and slope angle $\phi$. For the sake of convenience to compare with Prandtl's case, we show $u_{\max }$ in its dimensional form $u_{\max }^{*}$ and $h_{u}$ nondimensionalized by $(\nu / N)^{1 / 2}$. In case of Prandtl (infinite slope length and heat source), $u_{\max }^{*}$ is independent of $\phi$ (about $2.2 \mathrm{~m} / \mathrm{s}$ in the figure's case). $R^{1 / 4}$ means the half length of heating or cooling source nondimensoinalized by

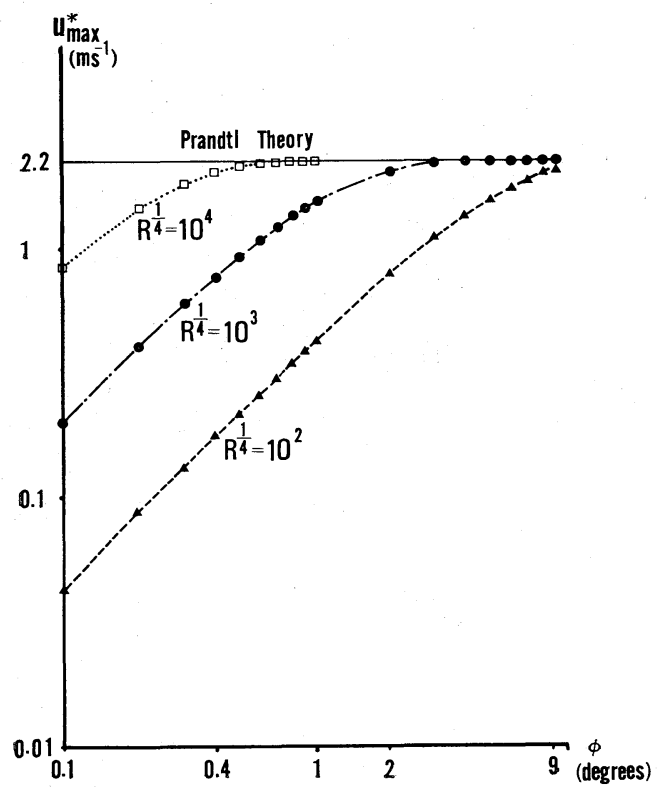

Fig. 4 The relation between maximum wind velocity $u_{\max }^{*}$ and slope angle $\phi . \quad u_{\max }^{*}$ 's are in dimensional form with $\nu=10 \mathrm{~m}^{2} \mathrm{~s}^{-1}$ and $N=1.96 \times 10^{-4} \mathrm{~S}^{-2}$

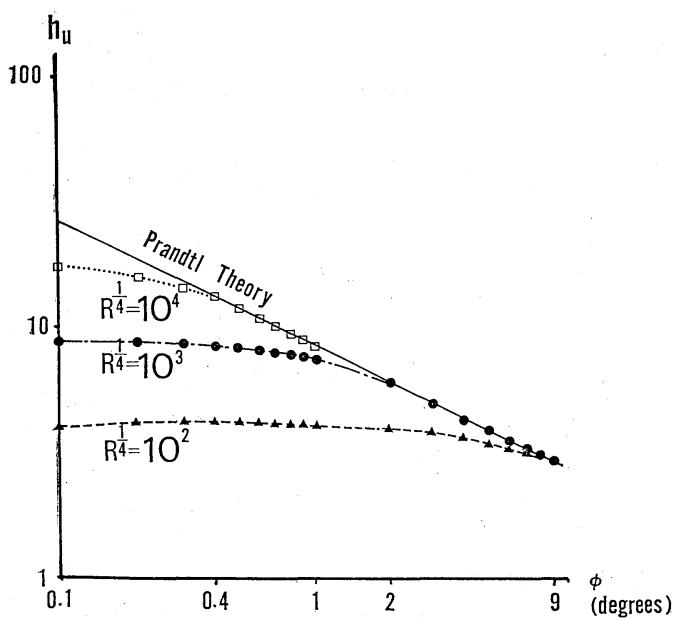

Fig. 5 The relation between $h_{u}$, the nondimensional height where $u_{\max }$ is given on the $\zeta$ axis, and $\phi$; To obtain dimensional height, multiply by $(\nu / N)^{1 / 2}$. $(\nu / N)^{1 / 2}$. When $\phi$ is small $u_{\max }^{*}$ 's are proportional to $\phi$, but at $\phi=0.4^{\circ}, \phi=2^{\circ}$ and $\phi=9^{\circ}$ $u_{\text {max }}^{*}$ 's of $R^{1 / 4}=10^{4}, R^{1 / 4}=10^{3}$ and $R^{1 / 4}=10^{2}$ cease to increase and become very close to Prandtl's case. Fig. 5 shows the dependency of the heights, where $u_{\max }^{*}$ 's are given, on $\phi$. In case of Prandtl this height $\left(h_{u}\right)$ is proportional to $(\sin \phi)^{-1 / 2}$ (c.f. equation $\left.(21)\right)$ so if $\phi$ is small $h_{u} \propto \phi^{-1 / 2}$. From Fig. 5 we see that these heights of $R^{1 / 4}=10^{4}, 10^{3}$ and $10^{2}$ are almost constants independent of $\phi$ until $\phi$ takes critical angles $\phi_{c}$ defined as $\phi_{c}=0.4^{\circ}$, $\phi_{c}=2^{\circ}$ and $\phi_{c}=9^{\circ}$ respectively, and after that they also approach Prandtl's case.

Next we consider what the critical angles $\phi_{c}=0.4^{\circ}, \phi_{c}=2^{\circ}$ and $\phi_{c}=9^{\circ}$ mean. Table 2 shows the relation between $h_{T}$ and $R^{1 / 4} \sin \phi$ on $R^{1 / 4}$ and $\phi_{c}$, respectively. Here $h_{T}$ is defined as the height, obtained by above linear theory and nondimensionalized by $(\nu / N)^{1 / 2}$, at which the temperature deviation on the $\zeta$ axis first become 0 over the heat island $(\phi=0)$ which has the same half length $\left(R^{1 / 4}\right)$ as the heat source on the slope. This parameter indicates the maximum height of the thermal boundary of the heat island (Fig. 6). Kimura (1975) showed $h_{T}^{*}=3.6 R^{-1 / 6} l$ in case of stationary heat island and this means $h_{T}^{*}=3.6(\nu / N)^{1 / 3} l^{1 / 3}$ in our case. From the relation between $R^{1 / 4}$ and $h_{T}$ in Table 2 we obtain $h_{T} \sim 3.5 R^{1 / 12}$ (The unit of $h_{T}$ is different from Kimura (1975).) and this become $h_{T}^{*}=3.5(\nu / N)^{1 / 3} l^{1 / 3}$. So our calculation agree well with Kimura in heat island case. From the same table we can also see $h_{T}$ is very close to $R^{1 / 4} \sin \phi_{c}$. This leads to the conclusion that the wind blows over the finite heat source on the slope behaves like slope wind (in Prandtl's sense ; Here after we call this Prandtl-like slope wind.) when a half of the height of the heat source $\left(R^{1 / 4} \sin \phi\right)$

Table 2 The relation among $R^{1 / 4}, 200 R^{-1 / 6}$, $3.5 R^{1 / 12}, \phi_{c}, h_{T}$ and $R^{1 / 4} \sin \phi_{c}$. We can find $\phi_{c} \sim 200 R^{-1 / 6}, h_{T} \sim 3.5 R^{1 / 12}$ and $h_{T} \sim R^{1 / 4} \sin \varphi_{c}$.

\begin{tabular}{c|c|c|c|c|c}
\hline$R^{1 / 4}$ & $200 R^{-1 / 6}$ & $3.5 R^{1 / 12}$ & $\phi_{c}$ & $h_{T}$ & $R^{1 / 4} \sin \phi_{c}$ \\
\hline $10^{2}$ & 9.3 & 16.2 & $9^{\circ}$ & 16.0 & 15.6 \\
$10^{3}$ & 2.0 & 35.0 & $2^{\circ}$ & 34.5 & 34.9 \\
$10^{4}$ & 0.4 & 75.4 & $0.4^{\circ}$ & 74.3 & 69.8 \\
\hline
\end{tabular}




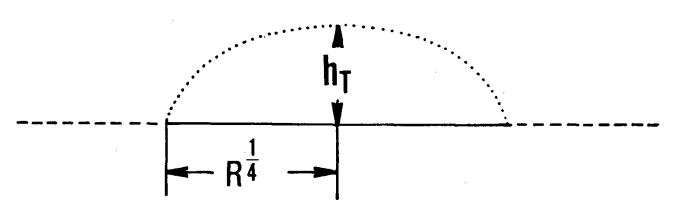

(a)

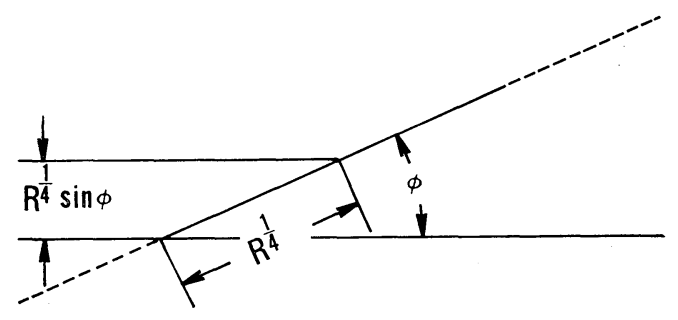

(b)

Fig. 6 (a) The depth of thermal boundary layer $\left(h_{T}\right)$ and (b) a half of the height of the slope $\left(R^{1 / 4} \sin \phi\right)$. When $h_{T}<R^{1 / 4} \sin \phi$ the phenomena are close to Prandtl's case. is much higher than the height of the thermal boundary layer which the heat island of same half length $\left(R^{1 / 4}\right)$ has.

\section{Numerical experiments}

In real case the situation is of course different from the linear theory. Since detailed observations of slope wind are not available, we will compare the results of linear theory with those of numerical experiments. Fig. 7 shows the results of 3-dimensional numerical calculation (Kondo, 1982, 1983) of local circulation in Harima area. These figures are cross sections from north to south through Himeji (the central city of this area). The configuration is relatively 2-dimensional around Himeji (Fig. 8) and mountain lies from east to west. The upper of the Fig. 7 is 1500 L.S.T. and the lower is 0400 L.S.T.. The diffusion coefficients of the numerical experiments are given
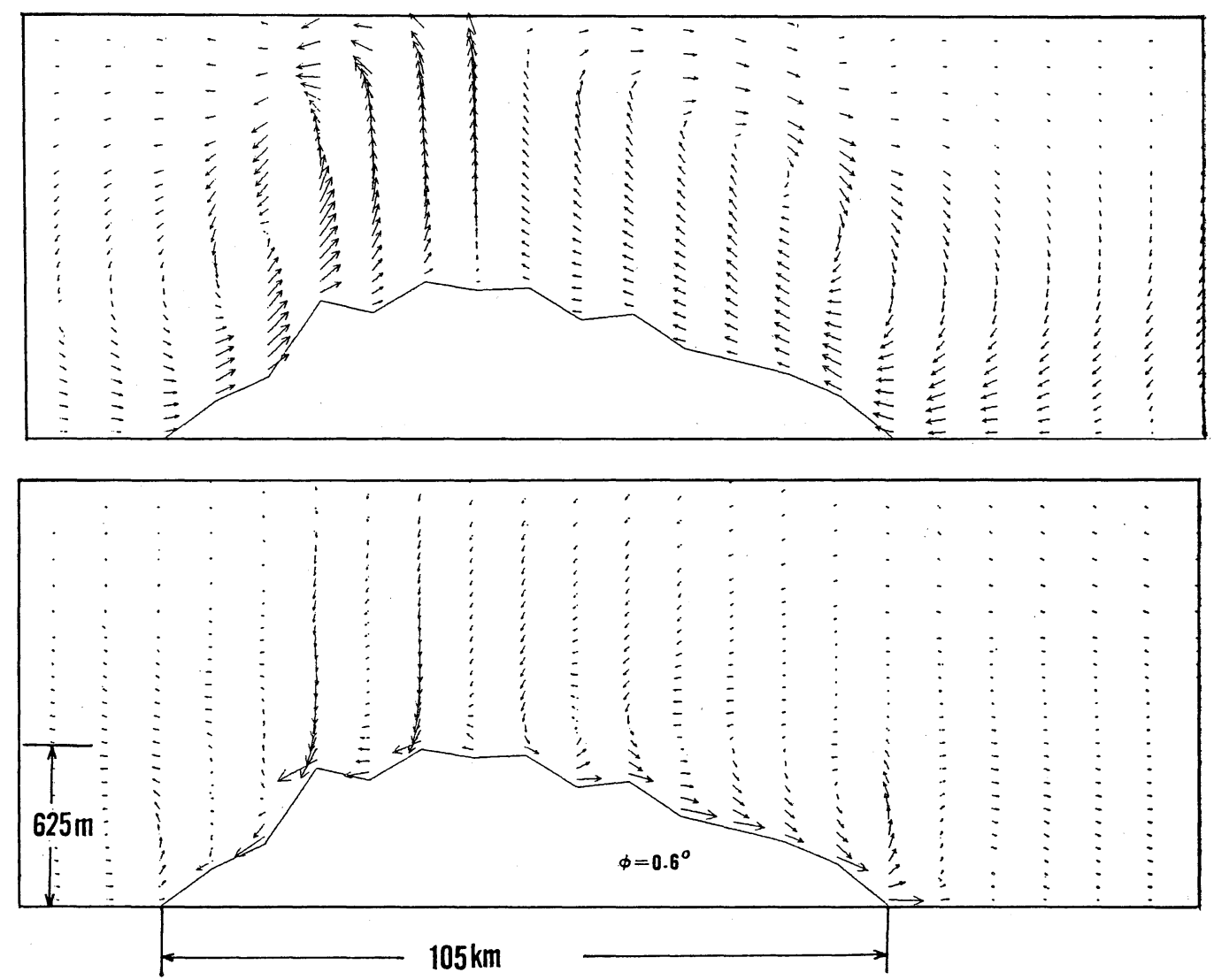

Fig. 7 Slope winds developed over Harima area resulting from numerical experiments. The upper is 1500 L.S.T. with $R^{1 / 4}=360$ and the lower is 0400 L.S. T. with $R^{1 / 4}=9500$. 


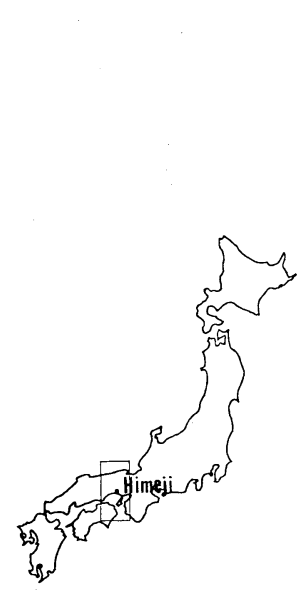

(A)

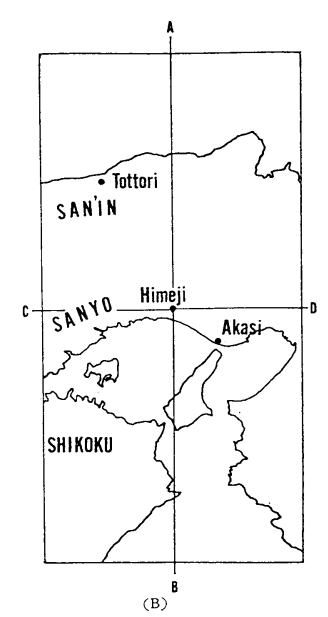

Fig. 8 The location of Harima and Himeji. Fig. 7 is a part of cross section of A-B (Left hand is north side in Fig. 7.).

by Gambo (1978),

$$
\begin{aligned}
& \left.\begin{array}{c}
K_{M}=l^{2}\left|\frac{\partial U}{\partial z}\right| f_{1}\left(R_{f}\right) \\
K_{\theta}=l^{2}\left|\frac{\partial U}{\partial z}\right| f_{2}\left(R_{f}\right)
\end{array}\right\} \text { for } R_{f}<R_{f c}=0.29, \\
& K_{M}=K_{\theta}=0.1 \mathrm{~m}^{2} / \mathrm{s} \quad \text { for } R_{f} \geq R_{f c}=0.29 \text {, }
\end{aligned}
$$

where $K_{M}$ is the diffusion coefficient of momentum and $K_{\theta}$ is that of potential temperature. They depend on $R_{f}$ (flux Richardson number); when $R_{f}>R_{f c}$ they bocome large but when $R_{f} \geq R_{f c}$ they are small constants.

From Fig. 7 we can see the difference of flow patterns between 1500 L.S.T. and 0400 L.S.T., for example, the thickness of the flows. So we investigate the difference using the results from above theory. We consider the average field between surface and $2000 \mathrm{~m}$ above.

In the daytime (1500 L.S.T.),

$$
\begin{aligned}
& \nu \cong 50 \mathrm{~m}^{2} \mathrm{~s}^{-1} \\
& N \cong 7.4 \times 10^{-3} \mathrm{~s}^{-1} \\
& l=30 \mathrm{~km} \\
& R^{1 / 4}=360
\end{aligned}
$$

at night (0400 L.S.T.),

$$
\begin{aligned}
& \nu \cong 0.1 \mathrm{~m}^{2} \mathrm{~s}^{-1} \\
& N \cong 1.0 \times 10^{-2} \mathrm{~s}^{-1}
\end{aligned}
$$

$l=30 \mathrm{~km}$

$$
R^{1 / 4}=9500
$$

and mean slope angle is $0.6^{\circ}$. From $h_{T}=$ $3.5 R^{1 / 12}$ we obtain $h_{T}=24.9$ (daytime) and $h_{T}=$ 74.1 (night) so that for the daytime we have $R^{1 / 4} \sin \phi=3.8<h_{T}=24.9$ and for night $R^{1 / 4} \sin \phi$ $=99.5>h_{T}=74.1$. Then in the daytime the flow is convective-slope wind but at night it is Prandtl-like slope wind. We may obtain same results from comparing $\phi$ with $\phi_{c}$. From Table 2 we can get $\phi_{c}\left({ }^{\circ}\right) \sim 200 R^{-1 / 6}$. This means $\phi_{c}=4.0^{\circ}$ in the daytime and $\phi_{c}=0.4^{\circ}$ at night in the case. So $\phi=0.6^{\circ}<\phi_{c}$ in the daytime then covective-slope wind becomes dominant and $\phi=0.6^{\circ}>\phi_{c}$ at night then Prandtl-like slope wind becomes dominant. These results are arranged in Table 3.

We can distinguish convective-slope wind from Prandtl-like slope wind by comparing $l \sin \phi$ with $h_{T}^{*}\left(h_{T}^{*}=3.5(\nu / N)^{1 / 3} l^{1 / 3}\right)$. Since only $N$ and $\nu$ can change between day and night and other parameters are constants, we can estimate the cause of these difference of slope wind. The ratio of $N^{-1}$ between day and

Table 3 The difference of parameters and characteristics of the flow between day and night.

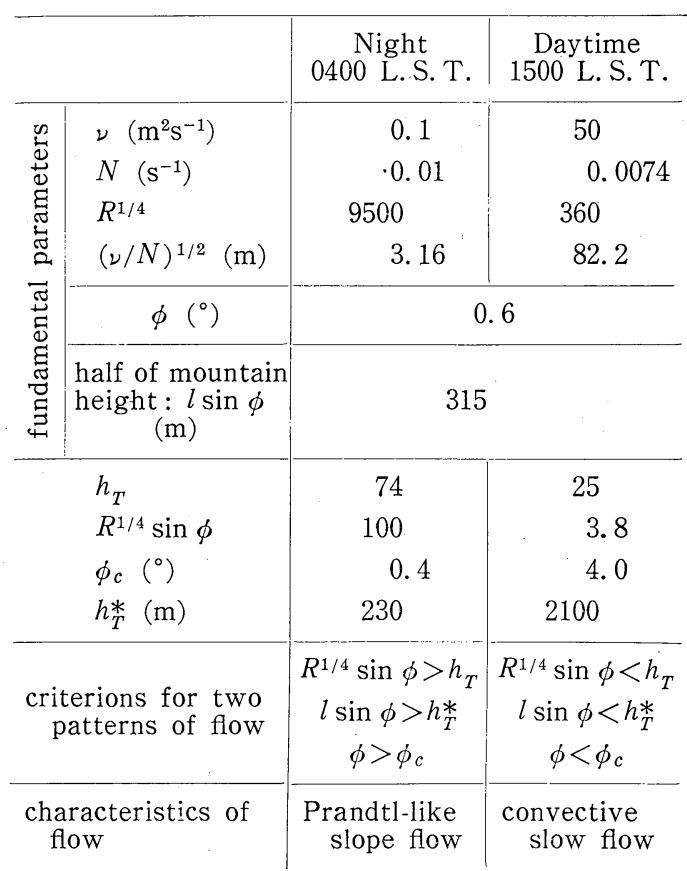


night $\left(N_{\text {day }} / N_{\text {night }}\right)^{-1}$ is 1.35 and this rather small but that of $\nu_{\text {day }} / \nu_{\text {night }}$ is 500 . From this we may conclude that the difference of eddy diffusivity between day and night makes the difference of the flow patterns of slope winds.

\section{Conclusions}

The flow pattern over an infinite slope in which finite length was heated or cooled was investigated by linear theory. When the slope slightly inclined, the flow pattern changed from heat island (two cells) to slope wind (one elongated cell). In the slope winds the slopeangle dependency of two parameters, the maximum wind velocity $u_{\max }^{*}$ and the height $h_{u}$, where $u_{\max }^{*}$ occured changed at the critical angle $\phi_{c}$ at which $l \sin \phi_{c}=h_{T}^{*}$. When a half of the slope height $l \sin \phi>h_{T}^{*}$, the height of thermal boundary layer over the heat island of length $2 l$, these parameters agreed well with the results obtained by Prandtl's theory.

The difference of slope flow between day and night obtained by numerical model was estimated by this criterion. In the daytime eddy diffusivity became large and $h_{T}^{*}$ was high so $l \sin \phi<h_{T}^{*}$ then the flow became convectiveslope wind. At night eddy diffusivity became small and $h_{T}^{*}$ became low so $l \sin \phi>h_{T}^{*}$ then the flow became Plandtl-like slope flow. This difference may be important when we consider the behavior of pollutants near the surface.

\section{Acknowledgement}

The author would express his sincere thanks to Dr. N. Yasuda, Tohoku University, Mr. H. Yoshikado, National Research Institute for Pollution and Resources, Dr. F. Fujibe, New Tokyo (Narita) Airport Local Meteorological Observatory, and Prof. K. Gambo, University of Tokyo, for fruitfull discussions and encouragements.

\section{References}

Asai, T. and S. Mitsumoto, 1978: Effects of an in- clined land surface on the land and sea breeze circulation: A numerical experiment. J. Meteor. Soc. of Japan, 56, 559-570.

Fujibe, F. and T. Asai, 1979: A study of local winds in Kanto District. Part 1: Structures of wind systems with diurnal variation (in Japanese). Tenki, 27, 595-604.

Gambo, K., 1978: Notes on the turbulence closure model for atmospheric boundary layers. J. Meteor. Soc. of Japan, 56, 466-480.

Kikuchi, Y., S. Arakawa, F. Kimura, K. Shirasaki and Y. Nagano, 1981: Numerical study on the effects of mountains on the land and sea breeze circulation in the Kanto District. J. Meteor. Soc. of Japan, 59, 723-738.

Kimura, R., 1975: Dynamics of steady convections over heat and cool islands. J. Meteor. Soc. of Japan, 53, 440-457.

Kimura, R. and T. Eguchi, 1978: On dynamical process of sea and land breeze circulation. $J$. Meteor. Soc. of Japan, 56, 67-85.

Kondo, H., 1981: A numerical experiment for the local circulation around Harima area: (1) Clear sky and no general wind case. Bull. Nat. Res. Inst. Pol. \& Res., 11, 17-30.

- 1983: A numerical experiment for the local circulation around Harima area: (2) An example of Lagrangian flow. Bull. Nat. Res. Inst. Pol. \& Res., 13, 75-83.

McHattie, L.B., 1968: Kananaskis valley winds in summer. J. Appl. Meteor., 7, 348-352.

Mannoji, N., 1982: A numerical experiment on the mountain and valley winds. J. Meteor. Soc. of Japan, 60, 1085-1105.

Oukouchi, Y., M. Uryu and R. Sawada, 1978: A numerical study on the effects of a mountain on the land and sea breezes. J. Meteor. Soc. of Japan, 56, 368-385.

Sahashi, K., 1981: Numerical experiment of land and sea breeze circulation with undulating orography part I, Model. J. Meteor. Soc. of Japan, 59, 361-372.

Stommel, H. and G. Veronis, 1957 : Steady convective motion in a horizontal layer of fluid heated uniformaly from above and cooled non-uniformaly from below. Tellus, 9, 401-407.

Sutton, O.G., 1953 : Micrometeorology, McGraw Hill, 323pp.

Tyson, P.D. and R.A. Preston-Whyte, 1972 : Observation of regional topographically-induced wind systems in Natal. J. Appl. Meteor., 11, 643-650. 


\title{
斜面流の昼と夜の差について
}

\author{
近 藤 裕 昭 \\ 通産省公害資源研究所
}

無限にのびる斜面のらちの有限長 $2 l$ を熱するか, あるいは冷却した場合の成層流体の応答を線型論により 調べた。斜面の角度が小さい場合には，斜面に近いところの流机の性質が斜面の高さの半分 $h_{S}^{*}=l \sin \phi(\phi$ は 斜面の角度）が同じ $2 l$ の長さを持つヒートアイランド上に発達する熱的境界層の高さ $h_{T}^{*}=\alpha(\nu / N)^{1 / 3} l^{1 / 3}$ ( $\alpha$ 〜3.5) よりも高いかどらかによって変化した。

実際の斜面に拈いては $h_{S}^{*}>h_{T}^{*}$ は夜間にはしばしば満たされるので, 流れはプラントルの理論の結果に近い

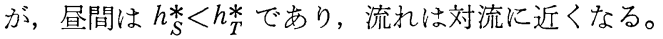

